
\title{
28 Research Suare \\ miR-4668-5p is up-regulated in serum of patients with pancreatic cancer
}

Pengfei Lv

Shanxi Cancer Hospital

Zhengwen Hao

Shanxi Cancer Hospital

Haoruo Zhang

Shanxi Cancer Hospital

Jun Li ( $\nabla 2684413031 @ q q . c o m$ )

Shanxi Cancer Hospital

Research

Keywords: miR-4668-5p; pancreatic cancer; upregulation; diagnosis

Posted Date: April 29th, 2020

DOI: https://doi.org/10.21203/rs.3.rs-25026/v1

License: (c) (1) This work is licensed under a Creative Commons Attribution 4.0 International License. Read Full License 


\section{Abstract \\ Backgroud:}

Pancreatic cancer is the most common of gastrointestinal tumor, with a high malignancy and poor prognosis,and early and effective diagnosis is the key for prolonging patient's overall survival, especially for fluid biopsy.

\section{Methods}

In this study, expression profile array was downloaded from GEO database, and differentially expressed miRNAs in serum were screened from patients with pancreatic cancer and healthy individuals, then coexpressed serum miRNAs in pancreatic cacner patients was analyzed by $\mathrm{R}$ software with weighted correlation network analysis(WGCNA) package. Finally, key differentially expressed miRNA was verified in serum of 16 patients with pancreatic cancer and 10 healthy individuals by quantitative real-time PCR (qRT-PCR).

\section{Results}

Results found that there were eleven differentially expressed miRNAs(miR-155-5p, miR-4668-5p, miR3613-3p, -miR-3201, miR-548ac, miR-486-5p, miR-548a-3p, miR-8084, miR-455-3p, miR-6068, and miR-

1246) between pancreatic cancer and healthy individuals. WGCNA results further found that miR-4668-5p has a higher associated modules compared to other differentially expressed miRNAs. Then, the expression level of miR-4668-5p was further verified, and results found that serum miR-4668-5p expression level was significantly higher in patients with pancreatic cancer than that of healthy individuals.

\section{Conclusion}

Our results firstly substantiated that miR-4668-5p was significantly up-regulated in serum of patients with pancreatic cancer, which may be a potential biomarker for pancreatic cancer diagnosis.

\section{Background}

With the development of socioeconomic and population ageing, pancreatic cancer is becoming the most common malignant tumor in China, and usually with a poor prognosis ${ }^{1}$. The most profound issue surrounding poor prognosis is wildly considered as lack of early diagnostic method and effective treatment ${ }^{2}$. 
In order to improve patients' prognosis, a majority of methods associated with diagnosis, therapy, and risk stratification are constantly exploring. Number of novel molecular biomarkers, associated with pancreatic cancer, was increasing gradually, including mutation status and abnormal expression of genes $^{3}$. Recently, DNA methylation ${ }^{4}$, cell-free $D_{N A}^{5}{ }^{5}$ exosomes $^{6}$ are becoming research hotpot. But there are very limited markers can be really used in clinic.

As we known, it is arduous to discover a novel biomarkers with high sensitivity and specificity ${ }^{7}$. Consequently, it is indispensable to explor more novel biomarkers associated with occurrence and development of pancreatic cancer. Here, a combination analysis of expression profile array and WGCNA was performed, which aimed to found and identify key molecular biomarkers related to pancreatic cancer diagnosis.

\section{Materials And Methods}

\section{Download and analysis of miRNAs expression profile.}

miRNAs expression profile array(GSE85589) was downloaded from GEO databse(https://www.ncbi.nlm.nih.gov/gds/), and serum miRNAs expression data was obtained from 88 patients with pancreatic cancer and 19 healthy control. Differentially expressed miRNAs were screened by $R$ software. Compared with healthy control, those miRNAs that absolute value of $\log _{2}$ FoldChange(FC) $>1$ and FDR $<0.05$ were considered as differentially expressed miRNAs.

\section{Weighted correlation network analysis(WGCNA) .}

Serum miRNAs expression data from 88 patients with pancreatic cancer was obtained from GSE85589, WGCNA analysis was performed according to previous report ${ }^{8}$.

\section{CDNA synthesis and quantitative real-time PCR .}

Serum( $2 \mathrm{ml}$ ) was collected from 10 healthy control and 16 pancreatic cancer patients from our hospital, and total RNA was extracted referring to previous report ${ }^{9}$. First-Strand cDNA synthesis kit (Agilent Technologies Inc.) was used for CDNA synthesis, and SYBR® Green PCR master mix (Applied Biosystems, NY, USA) was used to qRT-PCR. U6 as an internal control, and $1 \mu \mathrm{l}$ of a universal reverse primer (Agilent Technologies Inc., CA, USA) were used in this experiment. The relative miRNAs levels were determined in terms of their FC between patients with pancreatic cancer and healthy controls using $2^{-\Delta \Delta C t}$, and this experiment was performed in triplicate ${ }^{10}$. Primer sequence as follow:

miR-4668-5p:(F) AGGGAAAAAAAAAAGGATTTGTC;

U6:(F) CGCTTCGGCAGCACATATACTAA;

U6:(R) TATGGAACGCTTCACGAATTTGC. 


\section{Statistical analysis.}

IBM SPSS Statistics 21.0 software was used to statistical analysis. miR-4668-5p expression was compared between pancreatic cancer patients and healthy individuals via non-paired $t$ test. $P<0.05$ was considered as statistically significant.

\section{Results}

\section{Eleven dysregulated miRNAs were screened in serum of patients with pancreatic cancer.}

Serum miRNAs expression levels were compared between patients with pancreatic cancer $(n=88)$ and healthy controls $(n=19)($ Fig. $1 A)$, and eleven miRNAs were obviously dysregulated in serum of patients with pancreatic cancer, they are miR-155-5p, miR-4668-5p, miR-3613-3p, miR-3201, miR-548ac, miR-4865p, miR-548a-3p, miR-8084, miR-455-3p, miR-6068, and miR-1246. Among of them, miR-4668-5p, miR3613-3p, miR-3201, miR-548ac, miR-486-5p, miR-548a-3p, miR-8084, miR-6068, miR-1246 were upregulated in serum of patients with pancreatic cancer, and miR-155-5p and miR-455-3p were downregulated (FDR<0.05; Table 1).

miR-4668-5p was a key miRNA and associated with a multitude of modules. WGCNA package provides $R$ functions for weighted correlation network analysis, co-expression of miRNAs in serum of 88 patients were analyzed by R software, and eleven clusters (modules) of highly correlated genes were observed they were darkorange, salmon, darkmagenta, darkgrey, lightgreen, darkgreen, grey60, cyan, midnightblue, greenyellow, and lightcyan. Meanwhile, a heatmap of these miRNAs was shown (Fig. 1B-D).As shown in Table 2, differentially expressed miRNAs previous founded were almost included in darkgrey, darkmagenta and lightgreen modules. Then MM value of these miRNAs was calculated, highly correlated modules for these differentially expressed miRNAs were listed in Table $2(P<0.05)$, and we further observed that number of correlated modules of miR-4668-5p was highest among these miRNAs, including darkmagenta, darkgrey, lightgreen, grey60, and greenyellow. Hence, miR-4668-5p may be a key molecular biomarkers.

\section{miR-4668-5p was consistently up-regulated in serum of patients with pancreatic cancer.}

According to GEO and WGCNA analysis results, miR-4668-5p was considered as a potential key molecular in pancreatic cancer. Then, expression level of miR-4668-5p was further investigated by database and qRT-PCR assay. In GSE85589,up-regulated miR-4668-5p was observed in serum of patients with pancreatic cancer $(\mathrm{n}=88)$ compared to healthy control $(\mathrm{n}=19)(P<0.0001$;Fig. $2 \mathrm{~A})$. To further identify whether miR-4668-5p was also up-regulated in our serum sample, its expression was detected in patients and healthy individuals by qRT-PCR. And results suggested that miR-4668-5p expression was consistently up-regulated in serum of patients with pancreatic cancer $(n=16)$ compared to those healthy control $(n=$ 10) $(P<0.05$; Fig. 2B). 


\section{Discussion}

In this study, eleven differentially expressed miRNAs in serum of patients with pancreatic cancer were firstly screened by GEO database. Meanwhile, co-expressed miRNAs in serum of patients with pancreatic cancer was further analyzed by WGCNA package. Among these differentially expressed miRNAs, modules correlated with miR-4668-5p was higher than other miRNAs, which may indicate miR-4668-5p was a key molecular biomarkers. Then, up-regulated miR-4668-5p were further investigated in pancreatic cancer patients.

Recent studies indicated that patients with incidentally diagnosed pancreatic cancer have better prognosis than those with symptoms ${ }^{11}$, which explain the benefits of early diagnosis in improving prognosis. In many of the diagnosis methods, liquid biopsy is a potential non-invasive diagnosis method, and with convenient, economical, and minimally traumatic features ${ }^{12}$. To explore whether there were potential biomarkers existed inserum of patients with pancreatic cancer, non-coding RNA profile was download from GEO database, and followed by the screening of differentially expressed miRNAs, eleven differentially expressed miRNAs were observed. However, it is indispensable to further identify key miRNA among these differentially expressed miRNAs. Different from other studies, here, a combimation of weighted correlation network analysis (WGCNA) and GEO database was used to constructed coexpression network and modules, which aimed to increase our understanding of the host-pathogen relationship. WGCNA has been gradually used to construct coexpression network ${ }^{13}$, especially for miRNAs. As an example, hub miRNAs related to prognosis were identified in colorectal cancer (CRC) based on WGCNA analysis ${ }^{14,15}$. Here, WGCNA was used to constructed modules and co-expressed network,and combined with differentially expressed miRNAs, which indicated miR-4668-5p was a key molecular among these differentially expressed miRNAs.

In fact, dysregulated miR-4668-5p has been observed in blood and tissues of many diseases. For example, miR-4668-5p level was higher in serum of hepatocellular carcinoma patients compared to healthy control ${ }^{16}$. Similarly, dysregulated miR-4668-5p was also observed in gastric cancer tissues ${ }^{17}$, and dedifferentiated liposarcoma tissues ${ }^{18}$ compared to healthy controls. Not only in malignant tumor, dysregulated miR-4668-5p was also associated with nervous system diseases, for example $A D^{10}$ and mesial temporal lobe epilepsy with hippocampal sclerosis ${ }^{19}$. However, it is limit that studies related to miR-4668-5p in pancreatic cancer. In this study, we observed and confirmed miR-4668-5p was obviously up-regulated in serum of patients with pancreatic cancer. Moveover, a combination analysis of GEO and WGCNA suggested that miR-4668-5p may indicate a key molecular biomarker.

Nevertheless, there are still some limitations in this study. For example, it is better to combine miRNAs expression data with clinical data, such as overall survival, clinical stage, ect. Besides, the potential mechanism of miR-4668-5p regulating target genes and its functions should be deeply explored in future. At last, it is necessary to detect miR-4668-5p expression in a large number of samples. 


\section{Conclusions}

Our study firstly found that miR-4668-5p was a key differentially expressed miRNAs, and consistently upregulated in serum of patients with pancreatic cancer compared to healthy control, which may be a potential early diagnosis marker for pancreatic cancer.

\section{Declarations}

\section{Consent for publication}

We confirmed that all subjects participated in this study signed written informed consent for publication their data and relevant information.

\section{Acknowledgements}

Not applicable.

\section{Funding}

No funding was received.

\section{Authors' contributions}

$\mathrm{PL}$ performed the primary bioinformatics analysis, experiments, and writed the manuscript; $\mathrm{ZH}$ and $\mathrm{HZ}$ made substantial contributions to data analysis and technical support. JL reviewed and approved this manuscript.

\section{Ethics approval and consent to participate}

The study has been approved by the ethics committee of our hospital, and the patient signed the informed consent form.

\section{Competing interests}

The authors declare that they have no competing interests.

\section{Author information}

${ }^{1}$ Department of general surgery, Shanxi Tumor Hospital, No.3 new worker street, Xinghualing, Taiyuan, Shanxi 0300000,P.R. China

\section{Availability of data and materials}

The datasets used and/or analyzed during the current study available from the corresponding author on reasonable request. 


\section{References}

1. Jia X, Du P, Wu K, Xu Z, Fang J, Xu X, Lin K. Pancreatic Cancer Mortality in China: Characteristics and Prediction. Pancreas. 2018;47:233-7.

2. McGuigan A, Kelly P, Turkington RC, Jones C, Coleman HG, McCain RS. Pancreatic cancer: A review of clinical diagnosis, epidemiology, treatment and outcomes. World J Gastroenterol. 2018;24:484661.

3. Previdi MC, Carotenuto P, Zito D, Pandolfo R, Braconi C. Noncoding RNAs as novel biomarkers in pancreatic cancer: what do we know? Future Oncol. 2017;13:443-53.

4. Mishra NK, Guda C. Genome-wide DNA methylation analysis reveals molecular subtypes of pancreatic cancer. Oncotarget. 2017;8:28990-9012.

5. Vietsch EE, Graham GT, McCutcheon JN, Javaid A, Giaccone G, Marshall JL, Wellstein A. Circulating cell-free DNA mutation patterns in early and late stage colon and pancreatic cancer. Cancer Genet. 2017;218-219:39-50.

6. Zhao C, Gao F, Weng S, Liu Q. Pancreatic cancer and associated exosomes. Cancer Biomark. 2017;20:357-67.

7. Chu LC, Goggins MG, Fishman EK. Diagnosis and Detection of Pancreatic Cancer. Cancer J. 2017;23:333-42.

8. Langfelder P, Horvath S. WGCNA: an R package for weighted correlation network analysis. BMC Bioinformatics. 2008;9:559.

9. Ruan QF, Jiang MJ, Ye ZQ, Zhao CL, Xie WG. [Analysis of microRNA expression profile in serum of patients with electrical burn or thermal burn]. Zhonghua Shao Shang Za Zhi. 2017;33:37-42.

10. Kumar S, Vijayan M, Reddy PH. MicroRNA-455-3p as a potential peripheral biomarker for Alzheimer's disease. Hum Mol Genet. 2017;26:3808-22.

11. Zhou B, Xu JW, Cheng YG, Gao JY, Hu SY, Wang L, Zhan HX. Early detection of pancreatic cancer: Where are we now and where are we going? Int J Cancer. 2017;141:231-41.

12. Chu D, Park BH. Liquid biopsy: unlocking the potentials of cell-free DNA. Virchows Arch. 2017;471:147-54.

13. Zhai X, Xue Q, Liu Q, Guo Y, Chen Z. Colon cancer recurrenceassociated genes revealed by WGCNA coexpression network analysis. Mol Med Rep. 2017;16:6499-505.

14. Yepes S, Lopez R, Andrade RE, Rodriguez-Urrego PA, Lopez-Kleine L, Torres MM. Co-expressed miRNAs in gastric adenocarcinoma. Genomics. 2016;108:93-101.

15. Zhou XG, Huang XL, Liang SY, Tang SM, Wu SK, Huang TT, Mo ZN, Wang QY. Identifying miRNA and gene modules of colon cancer associated with pathological stage by weighted gene co-expression network analysis. Onco Targets Ther. 2018;11:2815-30.

16. Pascut D, Krmac H, Gilardi F, Patti R, Calligaris R, Croce LS, Tiribelli C. A comparative characterization of the circulating miRNome in whole blood and serum of HCC patients. Sci Rep. 2019;9:8265. 
17. Bibi F, Naseer MI, Alvi SA, Yasir M, Jiman-Fatani AA, Sawan A, Abuzenadah AM, Al-Qahtani MH, Azhar El. microRNA analysis of gastric cancer patients from Saudi Arabian population. BMC Genom. 2016;17:751.

18. Fricke A, Cimniak AFV, Ullrich PV, Becherer C, Bickert C, Pfeifer D, Heinz J, Stark GB, Bannasch H, Braig D, Eisenhardt SU. Whole blood miRNA expression analysis reveals miR-3613-3p as a potential biomarker for dedifferentiated liposarcoma. Cancer Biomark. 2018;22:199-207.

19. Yan S, Zhang H, Xie W, Meng F, Zhang K, Jiang Y, Zhang X, Zhang J. Altered microRNA profiles in plasma exosomes from mesial temporal lobe epilepsy with hippocampal sclerosis. Oncotarget. 2017;8:4136-46.

\section{Tables}

Table 1 Differentially expressed miRNAs from GEO database.

\begin{tabular}{cccccc}
\hline microRNAs & $\left|\mathrm{Log}_{2} \mathrm{FC}\right|$ & AveExpr & $\mathrm{t}$ & P.Value & adj.P.Val \\
\hline miR-155-5p\# & 1.08 & 0.85 & 8.86 & $1.55 \mathrm{E}-14$ & $4.00 \mathrm{E}-11$ \\
miR-4668-5p* & 3.46 & 6.48 & -8.15 & $6.05 \mathrm{E}-13$ & $7.80 \mathrm{E}-10$ \\
miR-3613-3p* & 3.63 & 6.58 & -8.07 & $9.27 \mathrm{E}-13$ & $7.96 \mathrm{E}-10$ \\
miR-3201* & 2.30 & 2.59 & -7.78 & $4.14 \mathrm{E}-12$ & $2.67 \mathrm{E}-09$ \\
miR-548ac* & 1.10 & 1.91 & -6.94 & $2.76 \mathrm{E}-10$ & $1.19 \mathrm{E}-07$ \\
miR-486-5p* & 1.04 & 6.62 & -6.39 & $4.08 \mathrm{E}-09$ & $1.17 \mathrm{E}-06$ \\
miR-548a-3p* & 1.02 & 2.09 & -6.21 & $9.44 \mathrm{E}-09$ & $2.18 \mathrm{E}-06$ \\
miR-8084* & 1.59 & 2.14 & -5.95 & $3.19 \mathrm{E}-08$ & $6.32 \mathrm{E}-06$ \\
miR-455-3p\# & 1.10 & 4.71 & 5.31 & $5.67 \mathrm{E}-07$ & $7.39 \mathrm{E}-05$ \\
miR-6068* & 1.00 & 3.02 & -4.78 & $5.44 \mathrm{E}-06$ & $4.38 \mathrm{E}-04$ \\
miR-1246* & 1.00 & 4.86 & 3.70 & $3.39 \mathrm{E}-04$ & $1.07 \mathrm{E}-02$ \\
\hline
\end{tabular}

*:up-regulation;\#:down-regulation;

Table 2. Models and MM value of these differentially miRNAs

\begin{tabular}{|c|c|c|c|c|c|c|}
\hline Model & miRNAs & & MM & 070.05 & & \\
\hline Darkgrey & $\begin{array}{l}\text { hsa-miR-4668-5p } \\
\text { hsa-miR-3613-3p } \\
\text { hsa-miR-6068 }\end{array}$ & $\begin{array}{l}\text { darkmagenta } \\
\text { darkmagenta } \\
\text { greenyellow }\end{array}$ & $\begin{array}{c}\text { darkgrey } \\
\text { darkgrey } \\
\text { grey60 }\end{array}$ & $\begin{array}{l}\text { lightgreen } \\
\text { lightgreen } \\
\text { lightgreen }\end{array}$ & $\begin{array}{c}\text { grey60 } \\
\text { greenyellow } \\
\text { darkgrey }\end{array}$ & greenyellow \\
\hline Darkmagenta & $\begin{array}{c}\text { hsa-miR-548ac } \\
\text { hsa-miR-3201 } \\
\text { hsa-miR-548a-3p } \\
\text { hsa-miR-8084 }\end{array}$ & $\begin{array}{l}\text { darkmagenta } \\
\text { darkmagenta } \\
\text { darkmagenta } \\
\text { darkmagenta }\end{array}$ & $\begin{array}{l}\text { darkgrey } \\
\text { cyan } \\
\text { darkgrey }\end{array}$ & cyan & & \\
\hline Lightgreen & hsa-miR-155-5p & lightgreen & & & & \\
\hline
\end{tabular}

\section{Figures}


A

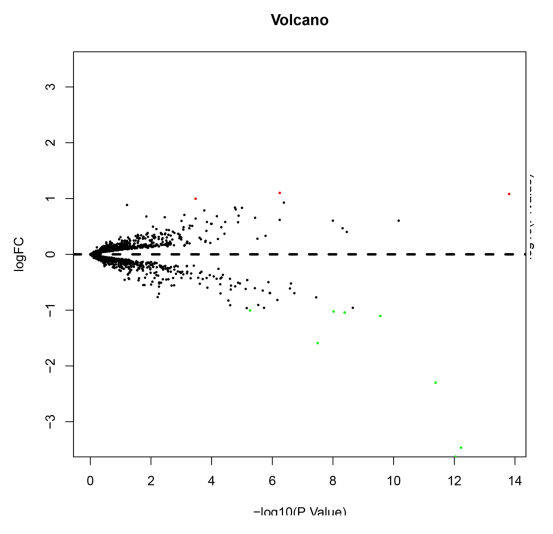

C

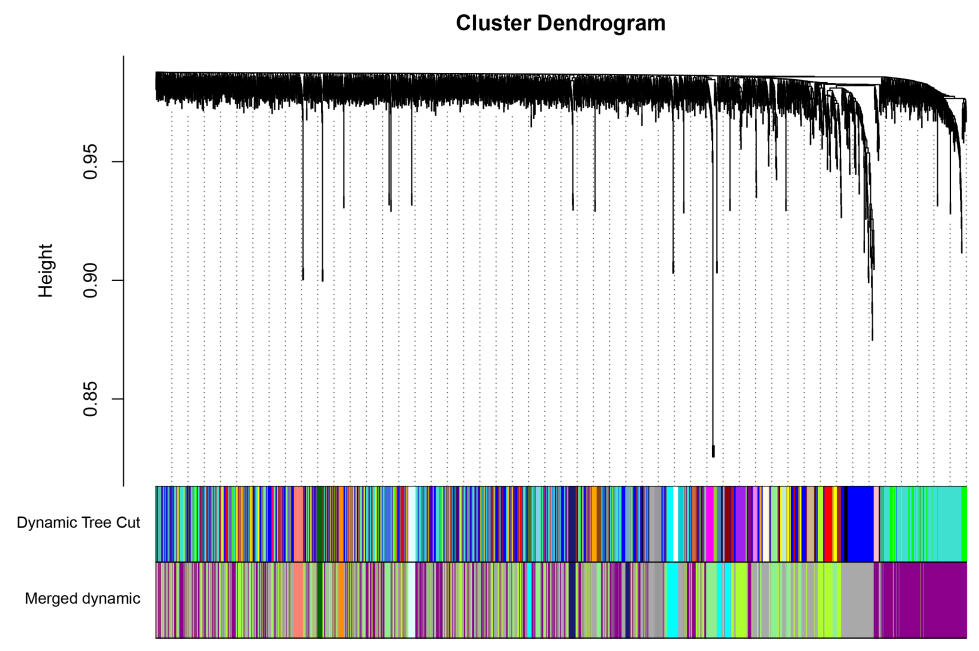

B

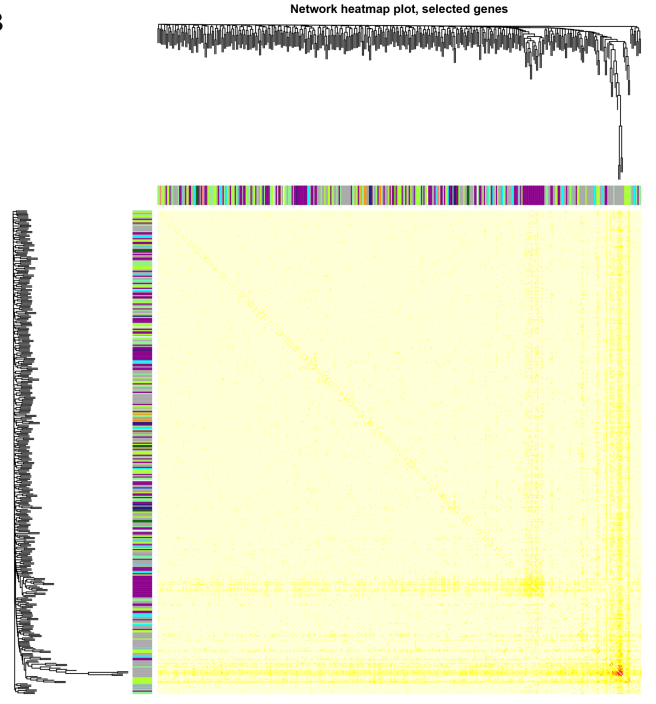

D

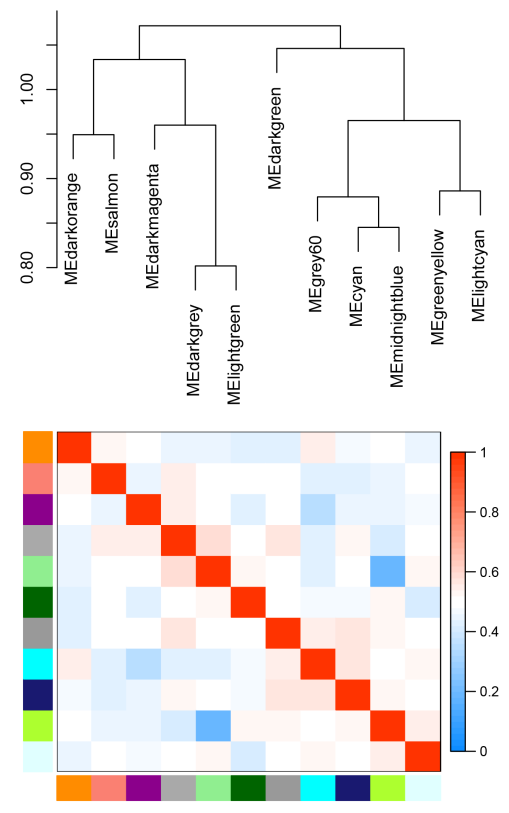

\section{Figure 1}

Combined screening of key differentially expressed miRNAs in serum between pancreatic and healthy control by GEO and WGCNA method. (A)Volcano map of differentially expressed miRNAs in serum of patients with pancreatic cancer from GEO database. (B) Heatmap of miRNAs expression in serum of patients with pancreatic cancer from GEO database. (C) Gene dendrogram obtained by average linkage 
hierarchical clustering. (D) Hierarchical clustering dendrogram of module eigengenes and heatmap plot of the adjacencies in the eigengene network. green: negative correlation; red: positive correlation.

A

miRNA-4668-5p

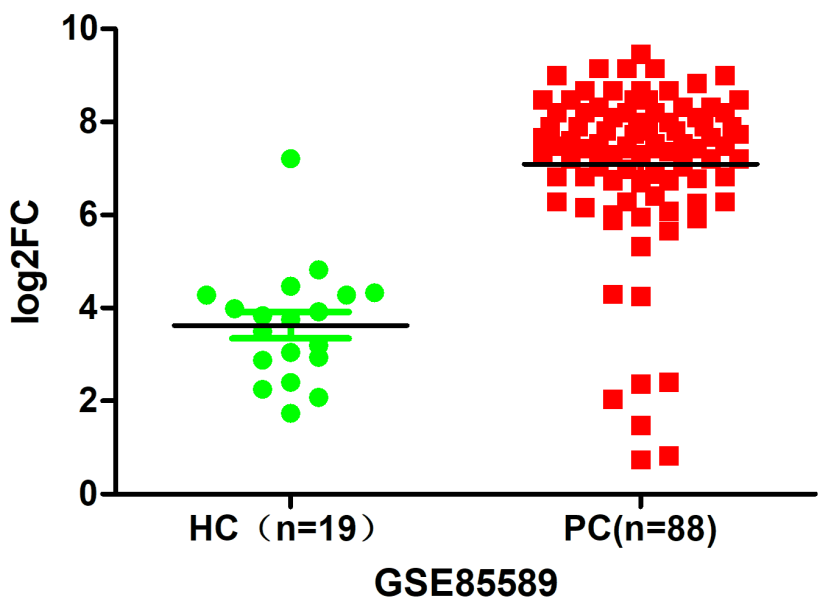

B

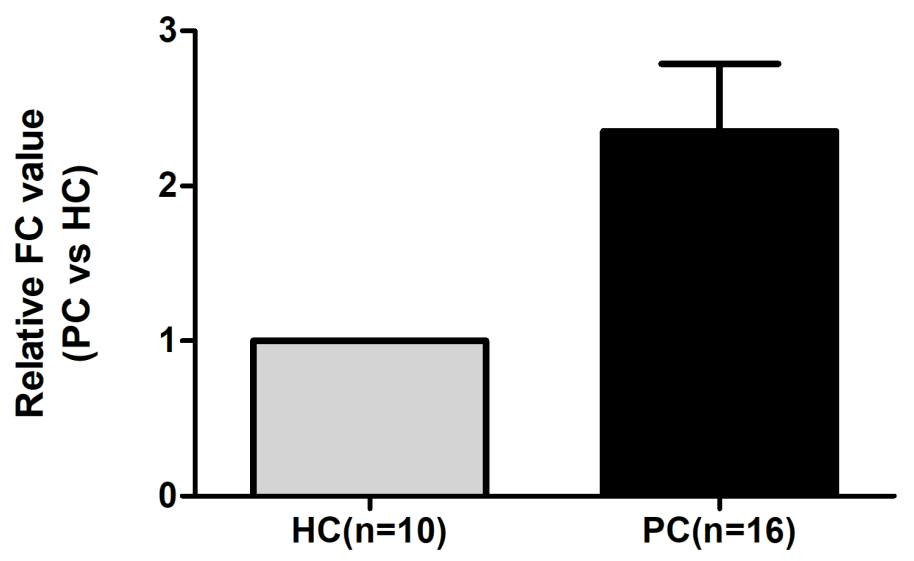

Figure 2

miR-4668-5p was significantly up-regulated in serum of patients with pancreatic cancer. (A) Up-regulated miR-4668-5p was observed in serum of patients with pancreatic cancer compared to healthy control based on GEO database. (B) Up-regulated miR-4668-5p was identified in serum of patients with pancreatic cancer compared to healthy control based on qRT-PCR method. HC: healthy control; PC: pancreatic cancer. 